\title{
Normalized Cuts for Spinal MRI Segmentation
}

\author{
Julio Carballido-Gamio ${ }^{1}$, Serge J. Belongie ${ }^{2}$, and Sharmila Majumdar ${ }^{1}$ \\ ${ }^{1}$ Magnetic Resonance Science Center, Department of Radiology, \#1 Irving Street, AC109, University of California, San \\ Francisco, San Francisco, CA, 94143-1290, USA \\ ${ }^{2}$ Department of Computer Science and Engineering, University of California, San Diego, CA, USA \\ \{jcarball, Sharmila.Majumdar\}@mrsc.ucsf.edu, sjb@cs.ucsd.edu
}

\begin{abstract}
Segmentation of bony structures plays an important role in image guided surgery of the spine. In this paper, we discuss a novel approach to the segmentation of vertebral bodies from 2D sagittal magnetic resonance images of the spine. We have applied Normalized Cuts with the Nyström approximation method to MR sagittal images of the spine taking windowed histograms of intensity or textons as the most promising features. Both, Normalized cuts and the Nyström approximation method have shown to be a powerful segmentation technique in different fields, but its usefulness in the medical imaging environment has not been shown yet. Results in T1 high-resolution images of the spine have been encouraging to pursue further research in this difficult task.
\end{abstract}

Keywords: Spine; vertebral bodies; magnetic resonance imaging (MRI); segmentation; Normalized cuts (Ncut); Nyström method.

\section{Introduction}

Physicians have commonly relied on computed tomography (CT) images to support their decisions in the diagnosis, treatment, and surgery of different pathologies of the spine due to the high resolution and good visualization of bone offered by this medical imaging modality. CT relies on the use of ionizing radiation, and does not depict soft tissue pathology, unlike magnetic resonance imaging (MRI). While the segmentation of vertebral bodies from CT images of the spine has commonly been accomplished with seed growing segmentation techniques, this task is more difficult in MRI, with variations in soft tissue contrast, and with the RF inhomogeneities, which increase the level of complexity.

In this paper we give a first approach to the segmentation of vertebral bodies from 2D sagittal MR images of the spine using Ncut as the segmentation technique with the Nyström method approximation. Ncut formulates segmentation as a graph-partitioning problem: it maximizes both the total dissimilarity between the different groups and the total similarity within the groups [1]. This segmentation technique readily admits combinations of different features such as brightness, position, windowed histograms, etc., thereby increasing its possible applications to different imaging modalities.

To alleviate the computational demand imposed by the Ncut technique, we employ the Nyström approximation method, which exploits the fact that there are fewer coherent groups than pixels in a typical image [2]. The Nyström approximation works by solving the grouping problem for a small subset of randomly chosen samples and then extending this solution to the complete set of samples. The application of the Nyström approximation to the solution of the eigenvalue problem of Ncut has also shown encouraging results in different areas than medical imaging.

\section{Materials and Methods}

\section{Magnetic Resonance Imaging}

Sagittal MR images of the spine of 2 patients were obtained at $1.5 \mathrm{~T}$ (Signa scanner; GE Medical Systems, Milwaukee, WI) using a surface coil. Patients were place supine in the MR scanner and T1 high-resolution images were acquired with the following parameters: $\mathrm{TR}=500 \mathrm{~ms}, \mathrm{TE}=14 \mathrm{~ms}$, flip angle $=90^{\circ}, \mathrm{FOV}=240$ x $240 \times 60 \mathrm{~mm}$, matrix size of 256x256x12 pixels, and slice thickness of $4.0 \mathrm{~mm}$ with a slice skip of 1.0.

\section{Segmentation of Vertebral Bodies}

In order to segment the vertebral bodies, we applied a coil correction algorithm based in low pass filtering to the volumes corresponding to the slices to be segmented.

Due to the fact that Ncut readily admits combinations of different features, we decided to apply this segmentation technique in two different ways. First, the features of interest were windowed histograms of intensity. Second, we create a set of features consisting of windowed histograms of textons [3], position and intensity. Although we performed this segmentation work in 2D, it is important to clarify that both, Ncut and 
the Nyström approximation method can be applied in 3D.

\section{- i) Windowed Histograms of Intensity}

The first step consists in the selection of the slice of interest, which is displayed to the user. The user then selects with a mouse click each vertebral body that she/he wants to segment. This information is used later after the segmentation procedure for displaying purposes.

A box of $5 \times 5$ pixels centered on each pixel is used to calculate local histograms of brightness with 20 bins equally spaced. A fixed value of 1 for the standard deviation $\left(\sigma_{\mathrm{HI}}^{2}\right)$ of this feature has shown to yield the best segmentation results.

In order to build the $\mathrm{W}$ matrix to solve the Ncut problem (e.g. the matrix containing the similarity weights between the different pixel features) through the Nyström approximation method, the $\chi^{2}$ test, a simple and effective measurement of histogram similarity [3], is performed based on the previous selection of 15 random histograms. Now we are ready to solve the eigenvalue problem of the Ncut technique through the Nyström approximation method [2]. This solution gives us as output 15 eigenvectors. The number of elements of these eigenvectors equals those of the original image, which means that each element of the eigenvectors can be mapped to one pixel in the image. As it is suggested in [2], K-means clustering algorithm is applied to the first eigenvectors corresponding to the smallest eigenvalues, with $\mathrm{K}(\mathrm{K}=3$ for windowed histograms of brightness) randomly initialized centers. In this paper we apply K-means function of the NETLAB toolbox [4].

The final step consists of a 4-connectivity labeling to the group containing the vertebral bodies selected by the user at the beginning in order to be displayed.

\section{- ii) Windowed Histograms of Textons}

The first step consists in the selection of the slice of interest, which is displayed to the user. The user then selects with a mouse click each vertebral body that she/he wants to segment. This information is used later after the segmentation procedure for displaying purposes.

The analysis of texture performed in this paper follows the guidelines proposed by Malik et al in [4] and [5]. Then a gaussian derivative filter set consisting of 2 phases (even and odd), 1 scale, and 5 orientations (equally spaced from 0 to $\pi$ ) is built as a difference-ofgaussian quadrature pair with 3:1 elongation. Also a second set of center-surround filters is built with 4 different scales. The filter bank consisting of a total of 14 filters (Figure 1), are $\mathrm{L}_{1}$ normalized for scale invariance [5] and then applied to the slice in the $\mathrm{k}$ space.

Each pixel of the image is now embedded in an $\mathrm{N}_{\mathrm{fr}}$ $=14$ dimensional vector of filter responses. These vectors are clustered with $K$-means into $K=4$ groups known as "textons", which in fact is a first segmentation of the image (Figure 2).

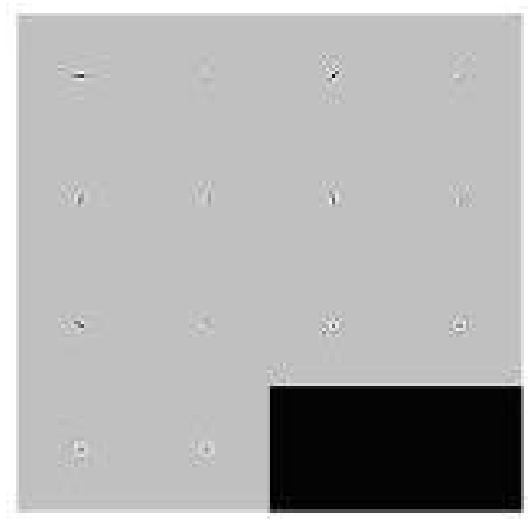

Figure 1. Filter bank consisting of gaussian derivative filters ( 2 phases, 1 scale, and 5 orientations), and centersurround filters (4 scales).

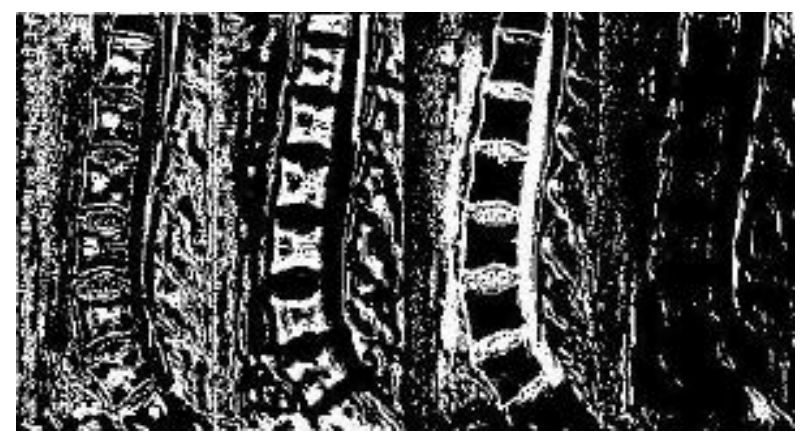

Figure 2. Mapping of pixels to their corresponding textons. (Images were cropped).

A box of $3 \times 3$ pixels centered on each pixel is used to calculate local histograms of textons with $\mathrm{K}=4$ bins. This can be written as [4]:

$$
\text { “ } h_{i}(k)=\sum_{j \in B(i)} I[T(j)=k]
$$

where $B(i)$ is the set of pixels in the box centered on $i$, $I[\cdot]$ is the indicator function, and $T(j)$ returns the texton assigned to pixel $j$." A $\sigma_{\mathrm{HT}}^{2}$ of 1 has shown to yield the best segmentation results for this feature.

A second three dimensional vector $(\mathrm{x}, \mathrm{y}, \mathrm{I})$ consisting of position $\left(\sigma_{P}^{2}=50000\right)$ and intensity $\left(\sigma_{I}^{2}=\right.$ 0.0001 ) is built for each pixel.

In order to build the $\mathrm{W}$ matrix to solve the Ncut problem through the Nyström approximation method, the $\chi^{2}$ test for histogram similarity, and $\mathrm{L}_{2}$ distance for $(\mathrm{x}, \mathrm{y}, \mathrm{I})$ vectors are performed based on the previous selection of 30 random samples. Features are combined 
and the solution gives us 30 eigenvectors, which the first of them corresponding to the smallest eigenvalues are clustered with $\mathrm{K}$-means $(\mathrm{K}=2)$ with randomly initialized centers.

The final step consists of a 4-connectivity labeling to the group containing the vertebral bodies selected by the user at the beginning in order to be displayed.

\section{Results}

We have produced encouraging results although we do believe that a final "accurate" segmentation will require the use of high-level features such as shape in the segmentation algorithm. Figure 3 shows results obtained from a single slice with both set of features, windowed histograms of brightness, and windowed histograms of textons with position and brightness.

Further improvements can be done to enhance the results shown in this paper. The most important are:

1. The algorithm performance degrades when we go far from the mid-sagittal slice. To improved this limitation, different approaches can be taken:

1.1 Correction of RF inhomogeneities as an image pre-processing step. A rapid and effective algorithm to deal with this problem has been suggested by Cohen et al in [6].

1.2 Application of the algorithm in 3D to increase the SNR, although it will require the acquisition of volumes with a slice skip of 0.0 for good performance.

1.3 Although the objective is the segmentation of vertebral bodies from images acquired with protocols that are well established such as T1 weighted, new MRI acquisition protocols that enhance the contrast between bone and soft tissue could certainly facilitate the segmentation task.

2. Time plays an important role in segmentation techniques based on spectral graph theories.

2.1 The Nyström approximation method alleviates this problem to a great extent. However, images such as those employed in medical imaging are considerably big. What we have done for this particular case is to crop the image to a region of interest of $81 \times 256$ pixels, which further improves the computational time.

2.2 The application of solutions as those described in 1 (above), and with prior knowledge of the different tissues that are present in the image, the number of samples required for the Nyström approximation method could be reduced. The user could select a fixed number of samples for each one of the different tissues composing the image and solve for them, instead of picking the samples randomly.

Although texture analysis has shown to yield good segmentation results, it is subtler to degrade in its performance, probably because texture in these images is not always well defined. However, it has opened the possibility for its application in different orthopaedic MRI applications, such as the segmentation of trabecular bone.

\section{Conclusions}

Accurate segmentation of vertebral bodies from MR images of the spine is still a task to be resolved. In this paper we have shown that Ncut combined with the Nyström approximation method provides promising segmentation results on MR images of the spine. We also have suggested different approaches that can be taken to improve the work presented in this paper.
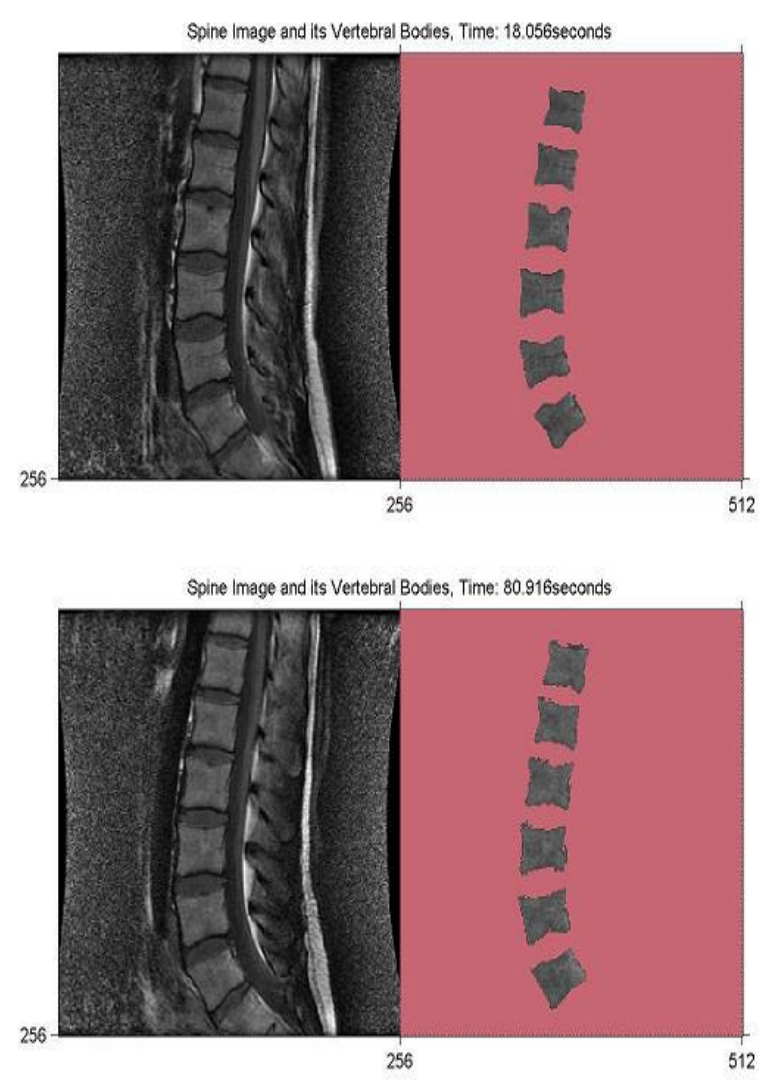

Figure 3. Results with windowed histograms of brightness (top), and windowed histograms of textons (bottom). The user did not select vertebral bodies at top and bottom of the images.

Use of different image data sets such as combination of $\mathrm{T} 1$ and $\mathrm{T} 2$ weighted images, combining CT and MR images and segmented volumes will provide improved specificity particularly in image guided surgery applications and the development of spinal models and atlases of spinal anatomy.

\section{Acknowledgements}


David Newitt. This work was support in part by a UCConacyt and Fulbright scholarships (to JCG).

\section{References}

[1] J. Shi and J. Malik. Ncut and image segmentation. IEEE Trans. PAMI, 22(8):888-905, August 2000.

[2] C. Fowlkes, S. Belongie and J. Malik. Efficient Spatiotemporal Grouping Using the Nyström Method. CVPR 2001, Kauai, HI.

Field Magnetic Resonance Imaging. Human Brain Mapping 10 (4), 2000.

[3] J. Malik, S. Belongie, J. Shi, and T. Leung. Textons, Contours and Regions: Cue Combination in Image Segmentation. Int. Conf. Computer Vision, Sept 1999, Corfu, Greece.

[4] I. Nabney and C. Bishop. Netleb neural network software. http://www.ncrg.aston.ac.uk/netlab/.

[5] J. Malik, S. Belongie, T. Leung and J. Shi. Contour and Texture Analysis for Image Segmentation. International Journal of Computer Vision, January 2000.

[6] Cohen MS, DuBois RD, Zeineh MM. Rapid and Effective Correction of RF Inhomogeneity for High Field Magnetic Resonance Imaging. Human Brain Mapping 10 (4), 2000. 